\title{
Small-angle X-ray scattering at the european synchrotron radiation facility
}

C. RIEKEL ${ }^{*}$, P. BÖSECKE* , O. DIAT ${ }^{*}$, P. ENGSTRÖM* ${ }^{*}$, M. KOCSIS ${ }^{*}$, S. LEQUIEN*, A. SNIGIREV ${ }^{* * *}$, I. SNIGIREVA ${ }^{*, * *}$ and K. TOTH*

${ }^{*}$ ESRF, BP. 220, 38043 Grenoble cedex, France

** Institute for Microelectronics Technology, Russian Academy of Sciences, 142432 Chernogolovka, Moscow Region, Russia

The status of small-angle scattering at the European Synchrotron Radiation Facility is reviewed. At the present stage three beamlines are foreseen. The high brilliance beamline will be used for time-resolved and ultralow-Q experiments. The anomalous dispersion beamline will serve principally the materials science community and allow edge studies from $\approx 2 \mathrm{keV}$ up to $\approx 35 \mathrm{keV}$. The microfocus beamline is actually in the commissioning phase. SAXS experiments have started on a classical mirror/monochromator camera. The presently reached beam size at the sample is $\approx 100^{*} 250 \mu \mathrm{m}^{2}$. Bragg-Fresnel focusing has been used to test a SAXS-camera with $a=2 * 2 \mu \mathrm{m}^{2}$ spot.

\section{Introduction}

The European Synchrotron Radiation Facility (ESRF) is a 3rd generation synchrotron radiation source (SR) which has come into operation in 1992. Brilliance and high photon energies are the main features of this $6 \mathrm{GeV}$ source1,2]. Table 1 gives an overview on the three SAXS/WAXS beamlines to be installed.

Table 1 ESRF-Beamlines to be used for small- and wide-angle scattering (SAXS/WAXS)

\begin{tabular}{|l|l|l|l|l|}
\hline BL-N & Name & Scientific Areas & Optimalisation & $\begin{array}{l}\text { User } \\
\text { Access }\end{array}$ \\
\hline BL\#1 & Microfocus & $\begin{array}{l}\text { Micro-Diffraction } \\
\text { Micro-Small-Angle Scattering }\end{array}$ & $\begin{array}{l}\text { small spot } \\
\text { flux density }\end{array}$ & 94 \\
BL\#4 (1) & High Brilliance & $\begin{array}{l}\text { a. Macromolecular Crystallography } \\
\text { on large Unit Cells } \\
\text { b. Real Time and Ultra Low-Q } \\
\text { SAXS }\end{array}$ & $\begin{array}{l}\text { small divergence } \\
\text { BL\#17 }\end{array}$ & $\begin{array}{l}\text { Anomalous } \\
\text { Scattering }\end{array}$ \\
\hline
\end{tabular}

\section{Source Properties}

The principal radiation sources of the ESRF are insertion devices such as undulators or wigglers. A rough distinction can be based on the $K$-value $(K=\alpha * \gamma)$ where $\gamma^{-1}$ is the opening angle of the photon emission cone of an electron ${ }^{(2)}$ and $\alpha$ the angle of the photon emission cone relative to the electron trajectory.[2] Undulator features with discrete line spectra are observed for $K \leqslant 3$ at the ESRF while an extended spectral range is observed in the wiggler-regime. An extended spectral range can also be covered with an undulator by "tuning" the magnetic field which implies a variation in $K$. Fig. 1 shows the comparison of the calculated and observed spectrum for the $K_{\max }=2.1$ undulator of the microfocus beamline. 


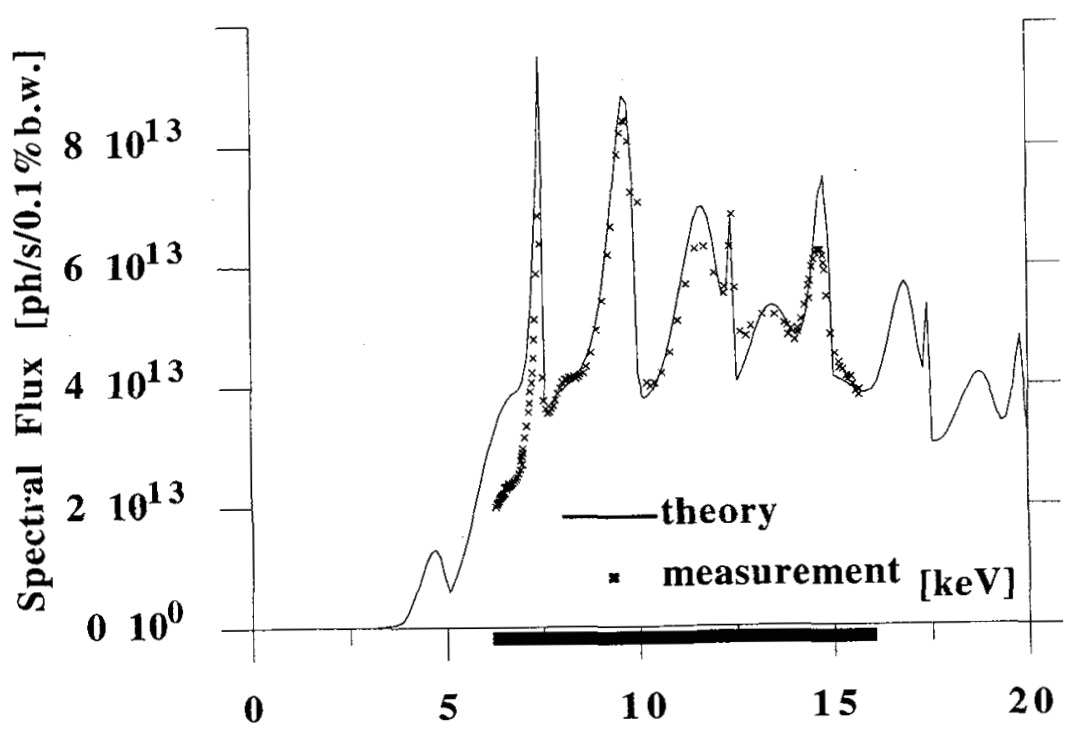

Fig.1 Calculated and measured spectrum of undulator of the microfocus beamline

Usually one can choose the period of the insertion device $-\lambda_{i}$ - and for undulators the location in a high- or low- $\beta$ section. A comparison of the photon source parameters for the insertion devices for the three SAXS-beamlines is given in Table 2 . Note that a high- $\beta$ section is optimized for small divergence while a low- $\beta$ section for small source size.

Table 2 Radiation sources for ESRF SAXS-beamlines; x:horizontal;z:vertical to the storage ring plane. Ring current: at $100 \mathrm{~mA}$

\begin{tabular}{|c|c|c|c|c|c|c|c|c|c|}
\hline$B L-N$ & Source & Location & $K_{\max }$ & $\begin{array}{c}\lambda_{u} \\
(\mathrm{~mm})\end{array}$ & $\begin{array}{l}\text { Power } \\
\text { (Watt) }\end{array}$ & $\begin{array}{c}x \\
(\mu \mathrm{m}-\end{array}$ & $\begin{array}{c}z \\
5 \sigma)\end{array}$ & $\begin{array}{c}x^{\prime} \\
(\mu \mathrm{rad} \\
\end{array}$ & $\begin{array}{c}z^{\prime} \\
5 \sigma \sigma) \\
\end{array}$ \\
\hline BL\#1 & undulator & low-B & 2.1 & 46 & $=200$ & 130 & 88 & 228 & 43 \\
\hline BL\#4 & undulator & high-B & $2.1(3)$ & 46 & $100^{(4)}$ & 1030 & 210 & 43 & 27 \\
\hline BL\#17 & wiggler & low- $\beta$ & 5 & 70 & $\approx 2100$ & 260 & 110 & 800 & 117 \\
\hline
\end{tabular}

\section{Instrumentation}

Bending magnet or wiggler sources have horizontal divergences up to a few mrad. For flux-optimized experiments the horizontal divergence is usually accepted by a focusing monochromator while the vertical divergence is accepted by a cylindrical or elliptical mirror $[3,4]$. For applications requiring energy tuning one may choose a double monochromator with a sagitally curved crystal for horizontal focusing $[5,6]$.

\footnotetext{
(3) a second segment with $\mathrm{K}_{\max }=0.5$ and $\lambda_{\mathrm{u}}=26 \mathrm{~mm}$ will be installed in order to increase the brilliance at $\approx 12 \mathrm{keV}$ (first harmonics) by $\approx$ factor 2 .

(4) central cone.
} 
Undulator sources have similar horizontal and vertical spot sizes at the optics. In this case one can accept the full beam divergence by mirror optics which may be either a combination of two orthogonal mirrors (Kirkpatrick-Baez geometry) or a double focusing mirror. This scheme has as additional advantage that it allows to separate focusing from monochromatisation.

\section{High-Brilliance Beamline}

The high brilliance beamline will make use of pinhole collimation. The optical system comprises a Si-111 channel-cut crystal and a toroidal mirror[7,8]. The monochromator allows to cover a wavelength range $2 \AA>\lambda>0.75 \AA$. SAXS-experiments at shorter wavelength than the often used $1.5 \AA$ are of interest for strongly absorbing samples, e.g. pressure cells. The main applications of BL\#4 will be in the area of timeresolved experiments up to the light scattering range. The close to 1:1 demagnification allows to preserve source divergence (Table 1.). The variation of the beam size with distance from the source is rather small as shown in Fig.2.

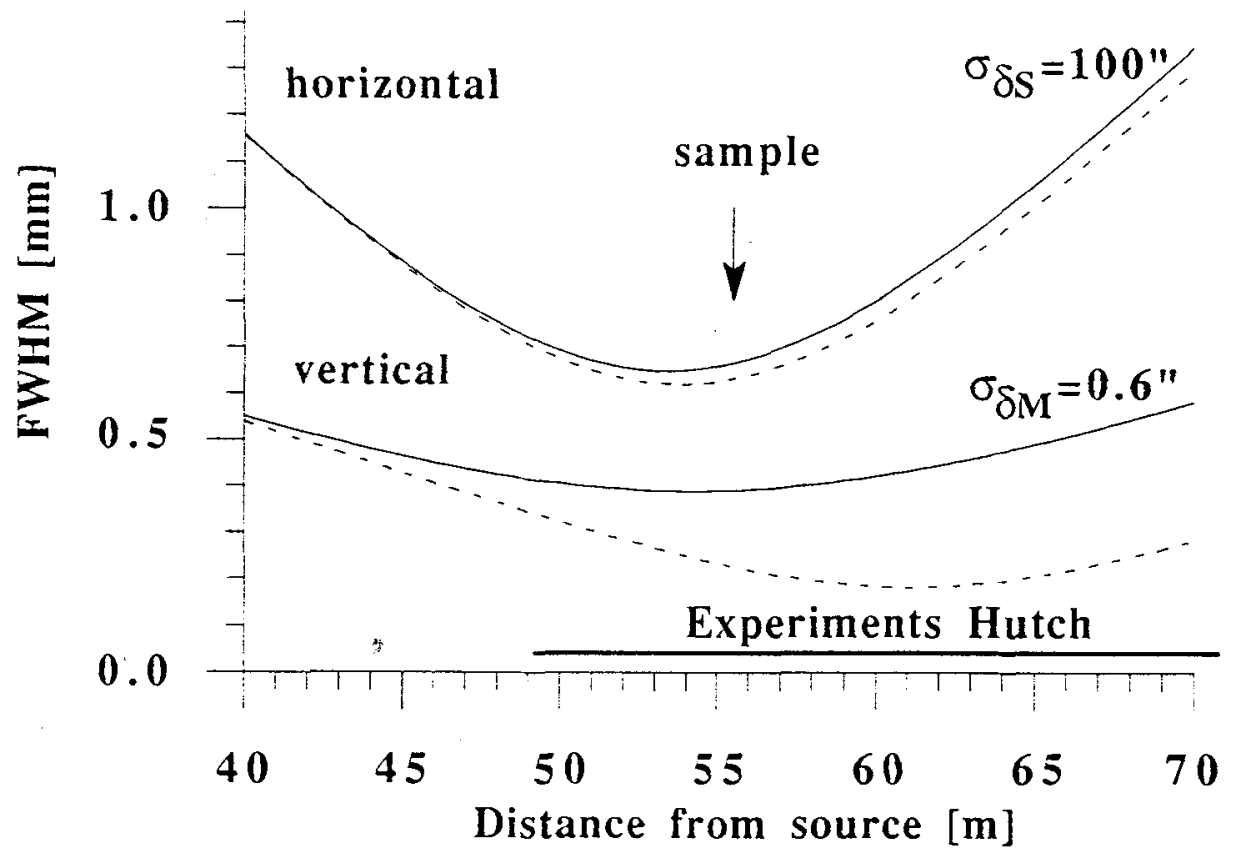

Fig.2 Variation of beamsize for high-brilliance beamline with sample to detector distance. Solid curves correspond to ideal mirror surfaces, barred curves assuming state-of-the-art surfaces.

In practice the detector will be moved inside a vacuum tube towards the sample. The variation of sample/detector distance from $0.5 \mathrm{~m}$ to $10 \mathrm{~m}$ allows an easy change of resolution conditions (Table 3 ). The minimum scattering vector $-Q_{\min }$ - has been calculated by considering pinhole collimation of the background [9]. The maximum scattering vector $-\mathrm{Q}_{\max }$ - is limited by the detector size. $\Delta \mathrm{Q}$ is the angular scattering resolution. The calculated flux at the sample position is $\approx 8^{*} 10^{12} \mathrm{Ph} / \mathrm{s}$ at $1 \AA$. In principle this value can be increased by at least an order of magnitude by asymmetrically cut Ge-crystals or multilayers [8]. The wavelength range of $2 \AA>\lambda>0.75 \AA$ is limited by the cutoff of the Rh-covered mirror $(\approx 0.6 \AA)$. 
Table 3: Calculated SAXS resolution. $Q=4 \pi \sin \Theta / \lambda, \lambda=1 \AA$, detector window: $200 \mathrm{~mm}$ diameter, $\mathrm{Q}_{\text {min }}, \mathrm{Q}_{\text {max }}$ : minimum and maximum scattering vector, $\Delta \mathrm{Q}$ : angular scattering resolution

\begin{tabular}{|ccccccc|}
\hline $\begin{array}{c}\text { distance between } \\
\text { sample and detector }[\mathrm{m}]\end{array}$ & $\begin{array}{c}\mathrm{Q}_{\mathrm{x}, \min }\left[\AA^{-1}\right] \\
0.50\end{array}$ & $\begin{array}{c}\mathrm{Q}_{\mathrm{x}, \max }\left[\AA^{-1}\right] \\
1.50\end{array}$ & $\begin{array}{c}\Delta \mathrm{Q}_{\mathrm{x}} \\
{\left[\AA^{-1}\right]}\end{array}$ & $\begin{array}{c}\mathrm{Q}_{\mathrm{z}, \min } \\
{\left[\AA^{-1}\right]}\end{array}$ & $\begin{array}{c}\mathrm{Q}_{\mathrm{z}, \max } \\
{\left[\AA^{-1}\right]}\end{array}$ & $\begin{array}{c}\Delta \mathrm{Q}_{\mathrm{z}} \\
{\left[\AA^{-1}\right]}\end{array}$ \\
4.00 & $9.4^{*} 10^{-3}$ & 1.3 & $8.8^{*} 10^{-3}$ & $5.2^{*} 10^{-3}$ & 1.3 & $5.0^{*} 10^{-3}$ \\
10.00 & $3.6^{*} 10^{-3}$ & $4.2^{*} 10^{-1}$ & $3.0^{*} 10^{-3}$ & $1.9^{*} 10^{-3}$ & $4.2^{*} 10^{-1}$ & $1.7^{*} 10^{-3}$ \\
& $1.8^{*} 10^{-3}$ & $1.6^{*} 10^{-1}$ & $1.3^{*} 10^{-3}$ & $8.8^{*} 10^{-4}$ & $1.6^{*} 10^{-1}$ & $6.9^{*} 10^{-4}$ \\
& $1.1^{*} 10^{-3}$ & $6.3 * 10^{-2}$ & $6.3 * 10^{-4}$ & $5.6 * 10^{-4}$ & $6.3^{*} 10^{-2}$ & $3.1 * 10^{-4}$ \\
\hline
\end{tabular}

\section{Anomalous Dispersion Beamline}

The applications of anomalous dispersion in materials research will be covered by a wiggler beamline which allows to extend the spectral range to $\approx 0.3 \AA$. An extension to the softer part of the spectrum $(\lambda \approx 6$ $\AA$ ) is envisaged in order to cover the K-absorption edges of biologically important elements such as $\mathrm{P}$ or $\mathrm{S}$. The choice of a wiggler with a low $\mathrm{K}$-value (Table 2.) allows to conserve brilliance as far as possible while ensuring a homogeneous spectral range. Furthermore the heatload on the first optical element can be reduced.

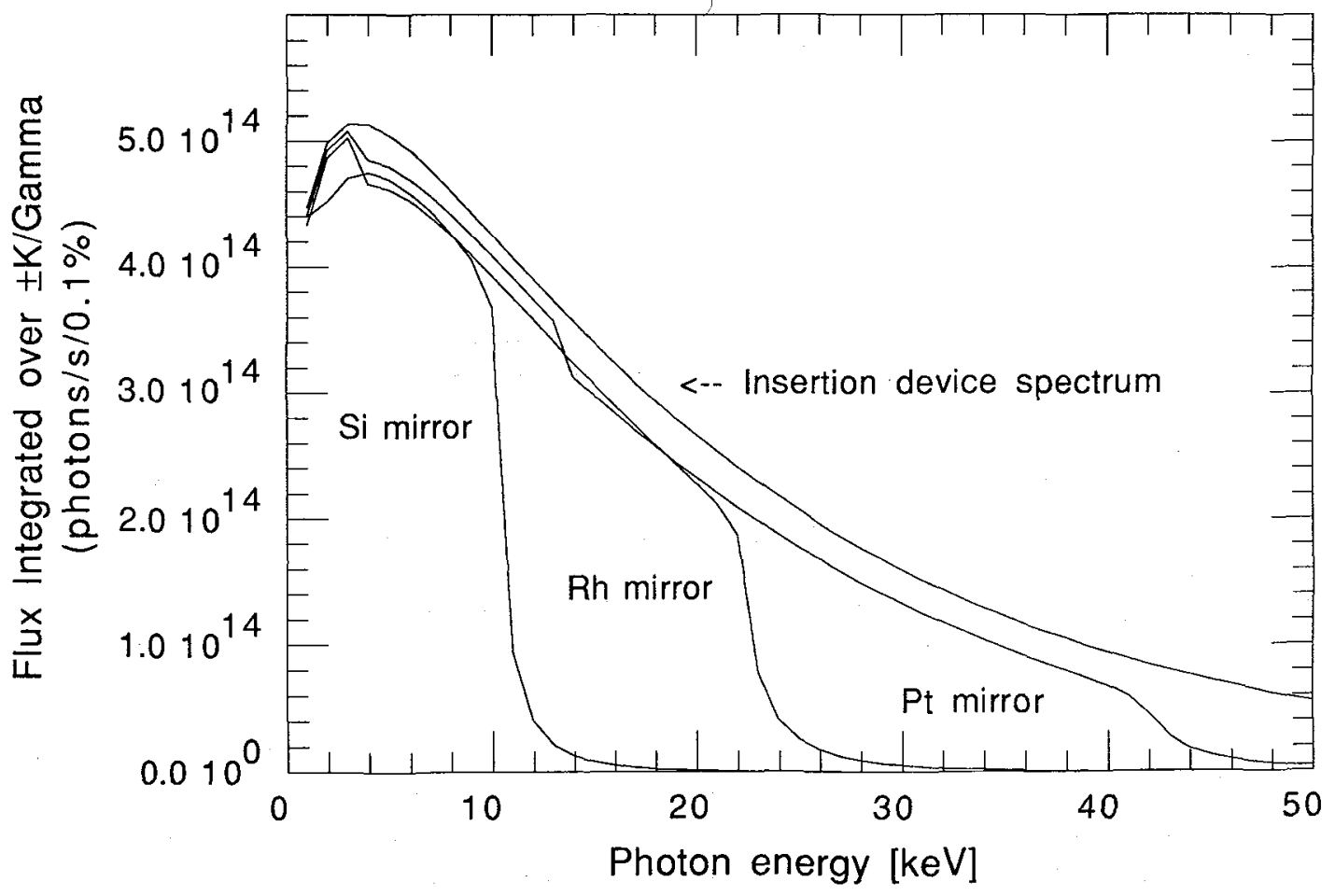

Fig.3 Spectral range and integrated flux of anomalous dispersion beamline[10]. Curves indicated correspond to direct wiggler beam on the pre-mirror and the beam after the pre-mirror. 
The full horizontal divergence of the source (Table 2.) can be accepted by a Si-monochromator with sagittal focusing. A pre-mirror is required to match the vertical divergence with the acceptance of the monochromator. Focalisation in the vertical plane is performed with a post-mirror made out of silicon. Both mirror surfaces will be separated into three stripes corresponding to (i) uncoated, (ii) coated with $\mathrm{Rh}$ and (iii) coated with $\mathrm{Pt}$ in order to be used for different energy ranges and to get a fixed-exit.(Fig.3) The spectral range covered is shown in Fig.3.[10] Ray-tracing suggests a focal spot of $\approx 100(\mathrm{~h}) * 200(\mathrm{v}) \mu \mathrm{m}^{2}$ with $4 * 10^{13} \mathrm{Ph} / \mathrm{s}$ which is of particular interest for selective area studies (e.g. single grains, surfaces and interfaces). The $\mathrm{Q}$-range of this beamline $\left(\mathrm{Q}_{\min } \approx 3^{*} 10^{-3} \AA^{-1}\right)$ is more limited than that of the high brilliance beamline in view of the overlap with other techniques (e.g. electron microscopy).

\section{Microfocus Beamline}

The microfocus beamline has been conceived principally for micro-diffraction and micro-SAXS.[8] and is one of the three test beamlines which has come into operation end of 1992 . The low- $\beta$ source point (Table 2.) will be demagnified by factor 10 by a double focusing ellipsoidal mirror.

The setup of the beamline has led to a separation of SAXS from diffraction. Thus a double focusing SAXS camera -derived derived from a laboratory camera [11]- has been installed in a separate experimental hutch at the end of the beamline. This camera uses the monochromatic beam from a channel-cut monochromator and focalizes the beam by a triangular Si-111 monochromator with $12^{\circ}$ asymmetric cut in the horizontal plane and a cylindrically bent Kanigen mirror in the vertical plane. Although still to be optimized, a spot size of $250(\mathrm{~h}) * 100(\mathrm{v}) \mu \mathrm{m}^{2}$ has already been observed at the sample position. The wavelength is fixed to $\approx 1.5 \AA(\mathrm{Si}-111)$ or $\approx 0.9 \AA(\mathrm{Si}-220)$ with a measured flux of $\approx 10^{11} \mathrm{Ph} / \mathrm{s}$ at $1.5 \AA$.

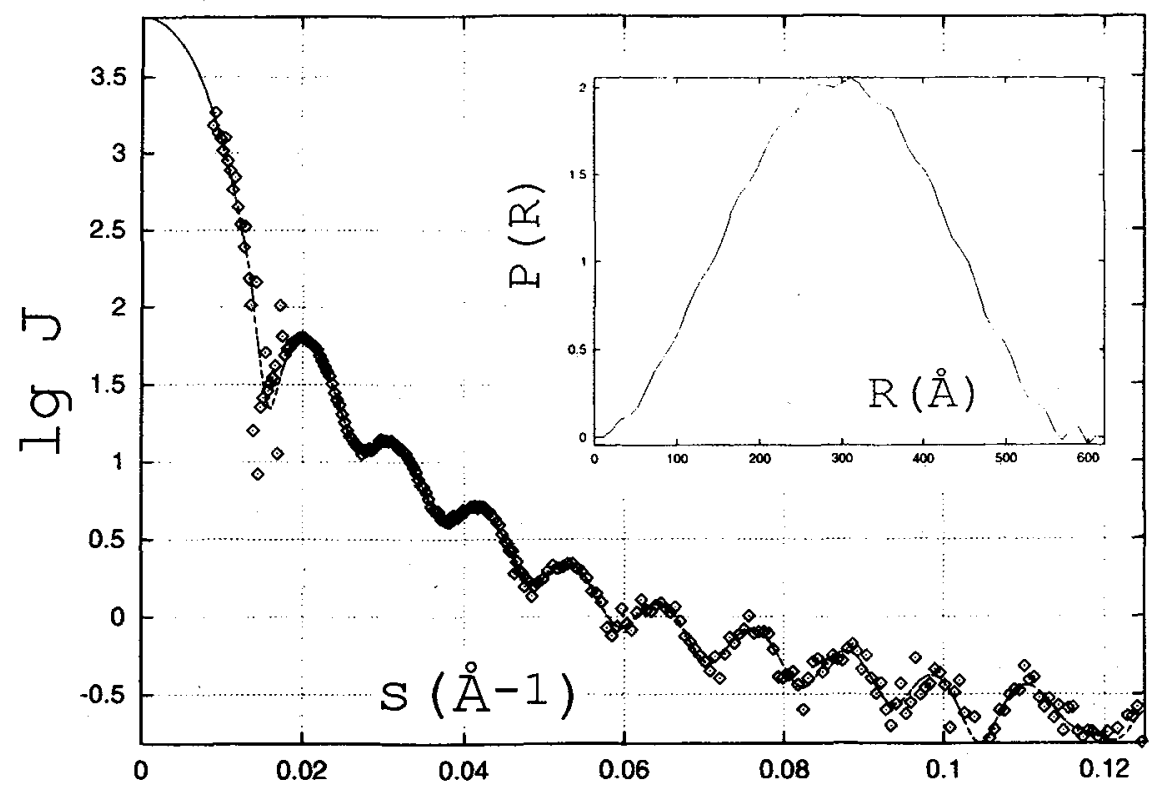

Fig.4 Radially averaged scattering pattern of $T 7$ bacteriophage in solution[12]. Inset shows particle distance distribution function. 
A small spot size is of interest for diffraction on small fibers and perturbation experiments on small sample volumes. Thus Fig.4 shows the scattering pattern of $\mathrm{T} 7$ bacteriophage in solution [12]. Apertures as small as $0.5 \mathrm{~mm}$ could be used in this case. For a concentration of $70 \mathrm{mg} / \mathrm{ml}$ the pattern was recorded in $2 \mathrm{~min}$ by a Molecular Dynamics image plate. The results compare very well to literature data[13] but extend over a larger Q-range .

Demagnifying mirror optics is limited at the microfocus beamline to $\approx 10 \mu \mathrm{m}$ focal spot size due to aberrations.[8] While multilayer mirrors might reach somewhat smaller spot sizes, different type of optics are required to reach the $\mu \mathrm{m}$ - or even sub- $\mu \mathrm{m}$-range. Candidates are phase-contrast Bragg-Fresnel[14] and tapered glass capillary optics[15,16]. Experience has been obtained for 2D-Bragg-Fresnel lens (BFL) focusing [17]. Thus Fig. 5 shows the pattern of native collagen which has been obtained with $a \approx 2 * 2 \mu \mathrm{m}^{2}$ focal spot (see inset) where the first order $(670 \AA)$ can be distinguished.[18] The limiting demagnification is determined by the outer fringe size which is $\Delta r_{n} \approx 0.4 \mu \mathrm{m}$ at present for a circular BFL. BFL-focusing is of particular interest for SAXS applications as the focal spot appears in a zone which is depleted from scattered radiation.(Fig.5)

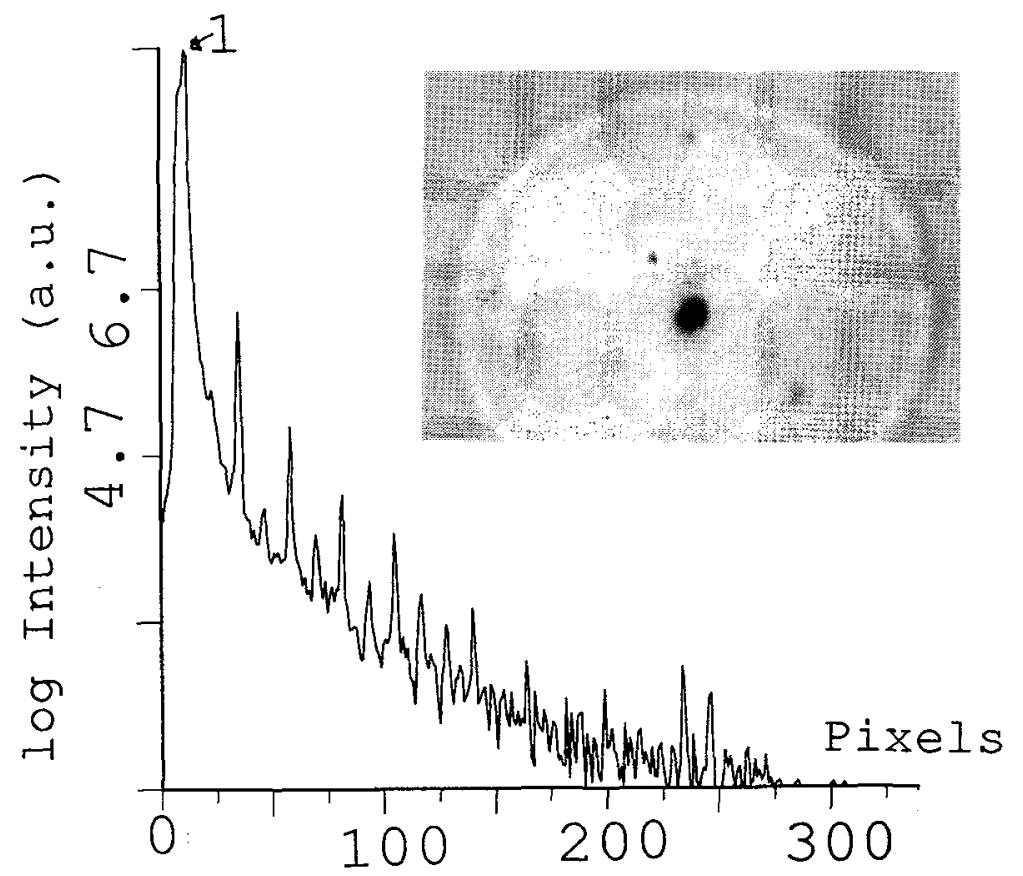

Fig.5 Radial cut of diffraction pattern of native collagen recorded by image plate.[18] First order peak indicated. Pixel size: $176 \mu \mathrm{m}$. Inset shows an optical microscopy image of the focus recorded on high density photographic paper. The diameter of the central spot is $\approx 2 \mu \mathrm{m}$.

The calculated total flux of a circular BFL with $200 \mu \mathrm{m}$ aperture is $\approx 10^{7} \mathrm{Ph} / \mathrm{s} / \mu \mathrm{m}^{2} / \mathrm{mA}$. Aperture size, efficiency $(\approx 30 \%)$ and bandwidth $\left(\triangle \mathrm{E} / \mathrm{E} \approx 10^{-5}\right)$ limit the overall flux of this optics as compared to the highbrilliance beamline at $1 \AA$ by factor $\approx 100$. Its resolution is limited to $\mathrm{Q}_{\min } \approx 2 * 10^{-3}$ due to the increase in divergence.

Capillary optics has yet to be tested for SAXS-applications. The extent to which the interior glass surface will increase the divergence of the beam and hence degrade the SAXS-resolution remains to be seen. The 
upper limit of divergence is given by the angle of total reflection which is $\approx 3 \mathrm{mrad}$ for glass at $1.5 \AA$. In contrast, the beam divergence $-\phi$-for a BFL-optics is given by the ration $\phi=A / F=\lambda / \Delta r_{n}$ where $A$ is the aperture of the BFL and $F$ its focal distance which is $\approx 0.3 \mathrm{mrad}$ for the $2 \mathrm{D}-\mathrm{BFL}$.

With the advent of microfocus SAXS-cameras, sample manipulation methods can be borrowed from electron microscopy. Thus Fig. 6 shows the location of a starch particle on an electron microscopy grid which allows to position the sample accurately in the photon-beam [19].

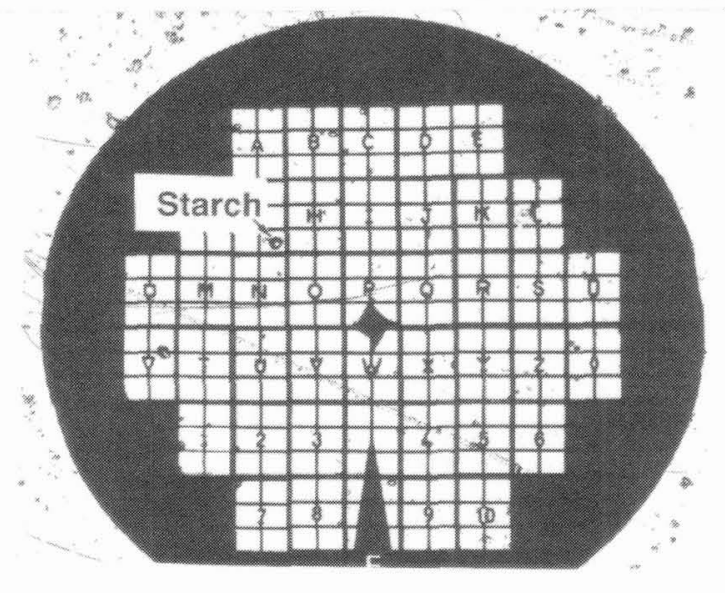

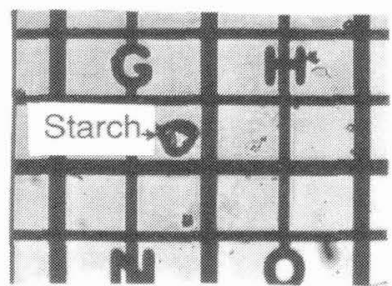

$135 \mu \mathrm{m}$

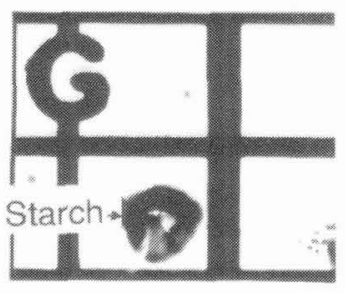

$135 \mu \mathrm{m}$

Fig.6 Optical micrograph of starch particle on electron microscopy grid.[19]

\section{Detection Systems}

The requirements for ms-readout for certain time resolved experiments (e.g. muscle scattering), a dynamic range of $\geq 10^{5}$, the lowest possible background noise and the possibility to record anisotropic scattering patterns favour 2D gas-filled detectors.

The approach taken at the ESRF is to redesign the readout part of the well established delay-line readout method[20,21] with VME/VXI components with the aim of anticipating possible future multiplexed detectors. Thus a new TDC[22] and a histogramming memory[23] have been developed and tested with a 2D gas-filled detector with a $20 \mathrm{~ns}$ delay-line. ${ }^{(5)}$ A counting rate of $\approx 5 \mathrm{MHz}$ for $1024 * 1024$ pixels is possible although the histogramming memory limits the system at present to $\approx 1.2 \mathrm{MHz}$. For this ultrafast delay-line the detector resolution is $\approx 0.9 \mathrm{~mm}$. An improvement of the resolution of the gas-filled detector will be necessary in particular for experiments at very small Q-values. As indicated above, off-line image

(5) the resolution can be as low as 20 ps depending on the choice of the clock speed 
plates are used at the ESRF for static SAXS-experiments. The use of image intensifiers for static or moderate time resolution experiments is an option for the future[24].

\section{Acknowledgements}

The supply of collagen samples by A. Miller and that of T7-bacteriophages by Gy. Rontó is gratefully acknowledged. $\mathrm{H}$. Chanzy helped with the development of small sample manipulation techniques. D. Svergun made the GNOM-package for the analysis of solution scattering data available.

\section{References}

[1] ESRF, Foundation Phase Report. 1987

[2] Riekel, C., in Synchrotron Radiation in Chemistry and Biology III, E. Mandelkow, Editor. 1989, Springer-Verlag: Berlin. 205-229

[3] Rosenbaum, G. and K.C. Holmes, in Synchrotron Radiation Research, S.D. H. Winick, Editor. 1980, Plenum Press: New York. 533-564

[4] Koch, M.H.J., Makrom. Chem. Macromol. Symp.,1988,15: 79-90

[5] Sparks, C.J., Borie, B.S., and J.B. Hastings, NIM, 1980,194: 73

[6] Krisch, M., Freund, A., Marot, G., Zhang, L., NIM, 1991,A305: 208-213

[7] Bösecke, P., Rev. Sci. Instrum., 1992,63(1):,438-441

[8] Riekel, C., P. Bösecke, and M.S.d. Rio,Rev. Sc. Instr., 1992,63(1): 974-981

[9] Elsner, G., C. Riekel, and H.G. Zachmann in Advances in Polymer Science, H.H. Kausch and H.G. Zachmann, Editor. 1985, Springer: Berlin

[10] Lequien, S., unpublished

[11] Riekel, C. and P. Suortti, J. Appl. Cryst., 1991,24: 549-554

[12] Toth, K., ESRF Internal Report,1993, EXP/KT/93/01

[13] Feigin, L.A., et al., NIM, 1989,A282: 488-489

[14] Aristov, V.V., et al., AIP Conf. Proc., 1986,147: 253-259

[15] Engström, P., et al., NIM, 1991,A302: 547-552

[16] Hoffman, S.A., Thiel, D.J., Bilderback, D.H., in Optics for High Brightness Synchrotron Radiation Beamlines. 1992,San Diego: SPIE.,252-257

[17] Basov, Y., et al., NIM, 1991. A308:,363

[18] Snigirev, A.,Snigireva,I., Riekel, C.,Miller, A., Wess, L., Wess, T., et al., this conference

[19] Chancy, H., Riekel, C., Snigireva, I., unpublished

[20] Hendrix, J., ed. Advances in Polymer Science, ed. H.H. Kausch and H.G. Zachmann. Vol. 67. 1985, Springer Verlag: Berlin. 59-98

[21] Boulin, C.J., Kempf, R., Gabriel, A., Koch, M.H.J., NIM, 1988,A269,312-320

[22] Berry, A., Rev. Sci. Instrum., 1993,64(5), in press;

[23] Barbosa, L., Boulin, C., Gabriel, A., Riekel, C., NIM, 1991,A302, 489-492

[24] Moy, J.P. and J. Morse. in Photoelectronic Image Devices 1991 - McGee Symposium Proceedings of the 10th Symposium. 1991, Imperial College, London: IOP Conference Series 